\title{
Article \\ Differential Immunostimulatory Effects of Lipoteichoic Acids Isolated from Four Strains of Lactiplantibacillus plantarum
}

\author{
Bong-Jun Jung ${ }^{1}$, Hangeun Kim ${ }^{2, *} \mathbb{D}$ and Dae-Kyun Chung ${ }^{1,2,3, *}$ \\ 1 Graduate School of Biotechnology, Kyung Hee University, Yongin 17104, Korea; bjjung@acebiome.com \\ 2 Research and Development Center, Skin Biotechnology Center Co., Ltd., Yongin 17104, Korea \\ 3 Skin Biotechnology Center, Kyung Hee University, Suwon 16229, Korea \\ * Correspondence: hkim93@khu.ac.kr (H.K.); dkchung@khu.ac.kr (D.-K.C.); \\ Tel.: +82-31-201-2465 (H.K. \& D.-K.C.); Fax: +82-31-202-3461 (D.-K.C.)
}

check for updates

Citation: Jung, B.-J.; Kim, H.; Chung, D.-K. Differential Immunostimulatory Effects of Lipoteichoic Acids Isolated from Four Strains of Lactiplantibacillus plantarum. Appl. Sci. 2022, 12, 954. https://doi.org/10.3390/app12030954

Academic Editors: Giovanna Donnarumma and Teresa Gervasi

Received: 9 December 2021

Accepted: 14 January 2022

Published: 18 January 2022

Publisher's Note: MDPI stays neutral with regard to jurisdictional claims in published maps and institutional affiliations.

Copyright: (c) 2022 by the authors. Licensee MDPI, Basel, Switzerland. This article is an open access article distributed under the terms and conditions of the Creative Commons Attribution (CC BY) license (https:// creativecommons.org/licenses/by/ $4.0 /)$.

\begin{abstract}
The intestinal health and immune modulatory effects of probiotics are well known. As with live bacteria, several studies demonstrating the ability of dead cells to improve gut health and immunity have suggested varying potentials for microbes to affect the human gut. The effect of dead microbes most likely derives from the cell wall of the microorganism. In this study, the functionality of lipoteichoic acid (LTA), a cell wall component, isolated from four stains of Lactiplantibacillus plantarum, $\mathrm{K} 8, \mathrm{~K} 88, \mathrm{~K} 5-5$, and $\mathrm{K} 55-5$, and the relationship between LTAs and their receptors were investigated. The four strains of L. plantarum have different LTA structures, which contributed to different immune activities in the immune cells. We confirmed that the different binding abilities with the host cell surface receptors, along with the differences in signal pathway, were due to the structural differences of the LTAs. LTA is an important postbiotic that induces various immunomodulatory actions.
\end{abstract}

Keywords: probiotics; lipoteichoic acid; Lactiplantibacillus plantarum; LTA structure; LTA-receptor affinity; postbiotics

\section{Introduction}

Lactiplantibacillus plantarum is a gram-positive bacterium and a well-known probiotic. Several strains of lactobacillus have been studied extensively for their benefits in gut health and immune regulation [1]. Cell wall components such as peptidoglycan (PGN) or lipoteichoic acid (LTA) have been shown to have an immunomodulatory effect [2]. LTA is composed of a poly (glycerophosphate) chain and glycolipids and is known to interact with Toll-like receptor (TLR) 2. The immune stimulation activities differ among bacterial species [3,4]. For example, LTA isolated from a pathogen such as Staphylococcus aureus increases the production of inflammatory cytokines and causes pulmonary inflammation and circulatory failure [5]. Conversely, LTA isolated from the cell wall of probiotics, such as lactobacillus, induces moderate inflammation, but strongly inhibits inflammation induced by pathogenic ligands, including LTA and lipopolysaccharide (LPS) [6,7].

Generally, LTA activates mitogen-activated protein kinase (MAPK) after interaction with TLR2, which initiates associations with myeloid differentiation primary response 88 (MYD88), interleukin-1 receptor (IL-1R)-associated kinases (IRAKs), and TNF-receptor-associated factor 6 (TRAF6) [8]. However, LTA seems to activate target genes using different signaling pathways. For example, serum response elements (SREs) in LTA-treated cells are regulated by the extracellular signal-regulated kinase (ERK) pathway, including the phosphorylation of ERK-1, MEK1/2, and c-Raf [9]. For induction of matrix metalloproteinase 9 (MMP-9), the PKC $\alpha$ dependent pathway, which is associated with activation of ATF2/AP-1 in RBA-1 cells, is activated by LTA [10]. In addition, LTAs isolated from different lactobacillus species have differing immune-regulatory effects, which are mediated by the MAPK signaling pathway [4]. The LTA-mediated inflammatory response requires LPS binding protein (LBP), CD14, and nuclear protein high-mobility group box 1 (HMGB1), as well as TLR2 interaction [11]. 
In the current study, LTAs were isolated from four strains of L. plantarum and tested for immunomodulatory activity. The binding affinity between LTAs and host cell surface receptors was investigated, and the structural differences among LTAs were analyzed.

\section{Materials and Methods}

\subsection{Bacteria Culture}

Four strains of L. plantarum [L. plantarum K8 (designated as K8), L. plantarum K88 (K88), L. plantarum K5-5 (K5-5), and L. plantarum K55-5 (K55-5)] were each inoculated in MRS media and incubated for $18 \mathrm{~h}$ in a $37^{\circ} \mathrm{C}$ incubator. The next day, the bacteria were transferred to fresh medium and cultured to the exponential phase $\left(\mathrm{OD}_{600} 1.0\right)$. The cells were harvested by centrifugation at $8000 \mathrm{rpm}$ for $10 \mathrm{~min}$ and washed 3 times with phosphate-buffered saline (PBS). The pellets were resuspended in PBS to the desired concentration.

\section{2. $16 S$ rRNA Sequencing}

16S rRNA analysis was performed for identification of the four strains of L. plantarum (Solgent Co., Ltd., Daejeon, Korea). Briefly, the four strains were streaked onto MRS agar plates and cultured, and single colonies were cultured in MRS broth and amplified. After that, gDNAs were extracted from the four strains by the boiling method using Chelex beads. After amplifying the $16 \mathrm{~S}$ rRNA by PCR using the primers indicated in Figure $1 \mathrm{~A}$, the nucleotide sequence was determined using BigDye ${ }^{\circledR}$ Terminator v3.1 cycle sequencing kits and an ABI3730XL DNA Analyzer. The phylogenetic tree was analyzed to determine the distance from the known L. plantarum strains.

A

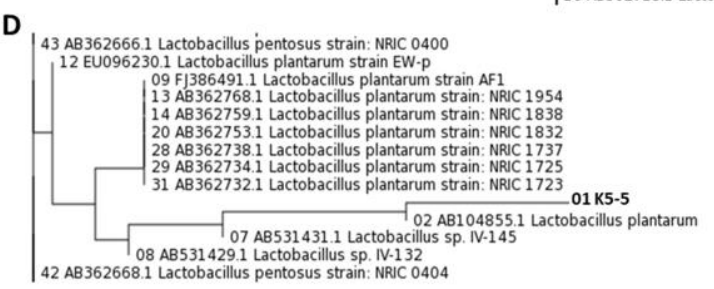
51 AB362655.1 Lactobacillus plantarum strain: NRIC 0386

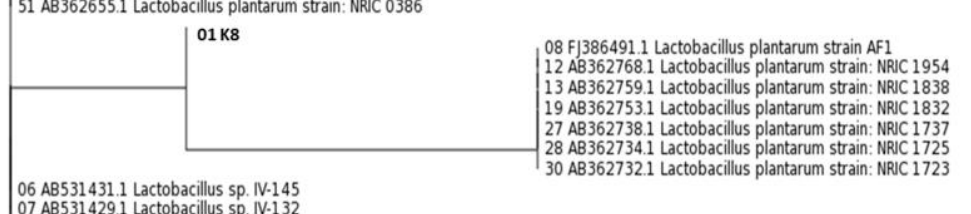

07 AB531429.1 Lactobacillus sp. IV.135 49 AB362658.1 Lactobacillus pentosus strain: NRIC 0391

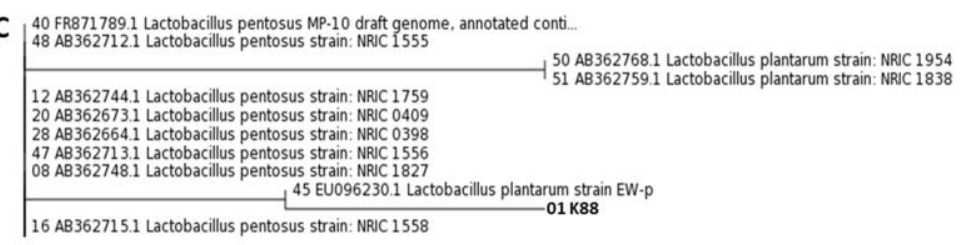

E

05 AB362755.1 Lactobacillus plantarum strain: NRIC 1834 04 AB362756.1 Lactobacillus pentosus strain: NRIC 1835 33 AB $01 \mathrm{~K} 55-5$

$01 \mathrm{~K} 55-5$
44 EU096230.1 Lactobacillus plantarum strain EW-p

43 EU825657.1 Lactobacillus plantarum

42 CP001617.1 Lactobacillus plantarum JDM1, complete genome

Figure 1. Identification of L. plantarum strains. (A) $16 \mathrm{~S}$ rRNA PCR products of K8, K88, K5-5, and K55-5 were loaded in 1\% agarose gel (upper panel). The $16 \mathrm{~S}$ rRNA primer set of L. plantarum is shown (lower panel). (B) Part of the phylogenetic tree for K8. (C) Part of the phylogenetic tree for K88. (D) Part of the phylogenetic tree for K5-5. (E) Part of the phylogenetic tree for K55-5.

\subsection{Cell Culture}

The human monocyte-like cell line THP-1 was maintained in RPMI-1640 medium supplemented with $10 \%$ fetal bovine serum (FBS), $2 \mathrm{mM}$ L-glutamine, $100 \mathrm{U} / \mathrm{mL}$ penicillin, and $100 \mu \mathrm{g} / \mathrm{mL}$ streptomycin in a humidified $37^{\circ} \mathrm{C}$ incubator with $5 \% \mathrm{CO}_{2}$. 


\subsection{Enzyme-Linked Immunosorbent Assay (ELISA)}

Supernatants were collected from THP- 1 cells treated with each of the four strains of L. plantarum. An ELISA microplate was coated with anti-TNF- $\alpha$ primary antibody (\#MAB610) and incubated overnight at $4{ }^{\circ} \mathrm{C}$. After incubation, the plate was washed 3 times with washing buffer $(1 \times$ PBS containing $0.05 \%$ Tween 20$)$. The microplate was blocked with blocking buffer ( $1 \%$ bovine serum albumin, $5 \%$ sucrose, and $0.01 \%$ sodium azide) for $1 \mathrm{~h}$ at room temperature (RT) and then washed 3 times. Culture supernatants were added to each well and incubated for $2 \mathrm{~h}$ at RT. The microplate was washed 3 times, and human TNF- $\alpha$ biotinylated detection antibody (\#BAF210) was added to each well and incubated for $2 \mathrm{~h}$. After washing 3 more times, horseradish peroxidase (HRP) substrate was added to each well. The reaction was stopped using stop solution and the absorbance was measured at $450 \mathrm{~nm}$ using a microplate reader. The antibodies used in this study were purchased from R\&D Systems (Minneapolis, MN, USA).

\subsection{Western Blot}

THP-1 cells treated with each of the four strains of L. plantarum were lysed with $2 \times$ sample buffer and separated in $12 \%(v / v)$ denaturing sodium dodecyl sulfate (SDS) gel. After transfer of the separated proteins to a polyvinylidene difluoride membrane, the membrane was pre-incubated in blocking buffer $(5 \%(w / v)$ non-fat dried milk in Trisbuffered saline with $0.1 \%(v / v)$ Tween 20 (TBS-T)) for $1 \mathrm{~h}$ at RT. Following blocking, the membranes were incubated with the primary antibodies anti-phospho-p38 (\#9211), antiphospho-JNK (\#9251), and anti-phospho-NFkB-p65 (\#3033) (Cell Signaling Technology, Danvers, MA, USA) for $2 \mathrm{~h}$ at RT. After the membranes were washed 3 times with TBS-T, they were incubated with HRP-conjugated secondary antibody for $2 \mathrm{~h}$. After washing 3 times with TBS-T, the protein bands were visualized using ECL Pico Western blotting reagent (Thermo Fisher Scientific Inc., Waltham, MA, USA) and detected with X-ray film. $\beta$-actin (sc-47778, Santa Cruz Biotechnology, Santa Cruz, CA, USA) was used as a loading control.

\subsection{Preparation of Lipoteichoic Acid}

LTAs were purified from the four strains of L. plantarum as previously described [6]. Silver staining and endotoxin assay (GenScript, Piscataway, NJ, USA) were performed to examine contamination with protein and endotoxin, respectively. We confirmed that there was no protein contamination and that the endotoxin contamination was less than $0.02 \mathrm{EU} / \mathrm{mL}$.

\subsection{Surface Plasmon Resonance (SPR) Analysis}

SPR was performed using the four LTAs and recombinant TLR2, TLR4, and CD14 proteins. SPR analysis was performed by Reichert Technologies ${ }^{\circledR}$ (Buffalo, NY, USA) using the Reichert SR7500DC system and Scrubber2 software.

\subsection{Analysis of LTA Glycolipids Structure}

Purified glycolipids from LTA were analyzed by GC/MS and LC-MS spectrometry as previously described [12]. All four strains of LTAs were subjected to alkali hydrolysis and methylation for GC/MS (Shimadzu, Kyoto, Japan) analysis. For each of the four samples, $1.5 \mathrm{mg}$ of sample was mixed with $0.5 \mathrm{~mL}$ of $1 \mathrm{M} \mathrm{KOH}$. The sample was put in a heating block and reacted at $80{ }^{\circ} \mathrm{C}$ for $30 \mathrm{~min}$. After cooling in an ice box, $0.5 \mathrm{~mL}$ of BF3 was added. The sample was placed in a heating block and reacted at $80^{\circ} \mathrm{C}$ for $30 \mathrm{~min}$, after which $1 \mathrm{~mL}$ of $\mathrm{n}$-hexane and $0.5 \mathrm{~mL}$ of $\mathrm{H}_{2} \mathrm{O}$ were added. After removing the upper layer (n-hexane layer) of the solution, $0.5 \mathrm{~mL}$ of $\mathrm{H}_{2} \mathrm{O}$ was added. After another decantation of the upper layer, $\mathrm{Na}_{2} \mathrm{SO}_{4}$ was added to remove moisture. GC/MS was measured after filtration using a syringe filter for the four alkaline hydrolysis and methylated samples. 


\subsection{Data Analysis}

Statistical analysis of the experimental data was performed using one-way analysis of variance (ANOVA), followed by Tukey's honestly significant difference (HSD) post hoc test and two-way ANOVA. The data shown are representative results of the mean \pm standard deviation (SD) of triplicate experiments. Differences were considered statistically significant when the $p$ value was $<0.05$. GraphPad Prism 5 software (GraphPad, San Diego, CA, USA) was used for the analysis.

\section{Results}

\subsection{Identification of the Four Strains of L. plantarum}

$16 \mathrm{~S}$ rRNAs from the four strains of L. plantarum were amplified by polymerase chain reaction (PCR) with the indicated primers (Figure 1A). To identify and classify the four strains of L. plantarum, the $16 \mathrm{~S}$ rRNA gene was sequenced and aligned using NCBI BLAST. The neighbor joining tree based on $16 \mathrm{~S}$ rRNA gene sequences shows the phylogenetic relationships between lactobacillus spp. and L. plantarum K8, L. plantarum K88, L. plantarum $\mathrm{K} 5-5$, or L. plantarum K55-5, with the bar indicating 0.02 substitutions per nucleotide position (Figure 1A-E). Bootstrap analysis was conducted to obtain confidence levels for the branches. All four strains used in the study were identified as L. plantarum species. The expanded phylogenetic tree is presented in Figure S1.

\subsection{The Four Strains of L. plantarum Showed Different Immune Activities}

THP-1 cells were treated with each of the four strains of L. plantarum for $18 \mathrm{~h}$, and the cytokine expression was examined with culture supernatants. As shown in Figure 2A, TNF$\alpha$ production was increased in a dose-dependent manner by all strains. In a quantitative comparison, K5-5 and K55-5 induced higher TNF- $\alpha$ expression than K8 and K88. In particular, K55-5 induced strong inflammation compared with other strains. Similar results were obtained with LTA isolated from the cell walls of the four strains of L. plantarum. LTAs isolated from the four L. plantarum strains induced the expression of TNF- $\alpha$ in a dosedependent manner. LTA isolated from K5-5 and K55-5 showed stronger TNF- $\alpha$ expression than that isolated from K8 and K88 (Figure 2B). This difference in immune busting might be due to variations in signaling activation. A representative signaling pathway activated by LTA was examined. After treating THP- 1 cells with LTAs at $100 \mu \mathrm{g} / \mathrm{mL}$ each, NF-kB p65 subunit, p38, and JNK signal activities were examined by Western blot. As expected, K5-5 and K55-5 induced phosphorylation of NF-KB-p65, p-38, and JNK, while K8 and K88 moderately induced signaling activation (Figure 2C). Densitometry data generated for Western blots showed that the protein abundance of K8 and K88 was lower than that of K5-5 and K55-5 (Figure 2D).

\subsection{Interaction Analysis of LTAs with Cell Surface Receptors}

SPR analysis was used to examine the associations between LTAs and their receptors. The binding reaction between one molecule of analyte and one molecule of receptor can be confirmed through analysis of the association and dissociation sections between LTAs and receptors such as TLR 2, TLR4, and CD14. The binding force between LTA and the receptor is determined by the association rate constant $(\mathrm{Ka})$ and the dissociation rate constant $(\mathrm{Kd})$. A lower Ka value indicates that the binding is stronger and faster, while a lower Kd value indicates fast dissociation. Lastly, KD is an indicator of affinity, and the lower index value suggests that the affinity is strong. Affinity to TLR2 of LPS and LTAs isolated from the four L. plantarum strains was analyzed (Table 1 and Figure S2). The Ka value was highest in K8 at $9.50 \times 10^{3}$ and decreased in the order of K88, K5-5, and K55-5. On the other hand, the $\mathrm{Kd}$ values were in the range of $5.02 \times 10^{-3}-5.9 \times 10^{-4}$, showing similar values. As a result, based on KD values, the binding affinity of LTA isolated from K5-5 and K55-5 to TLR2 was higher than that of LTA isolated from K8 and K88. The KD to TLR4 was highest in LPS and LTA isolated from K55-5. On the other hand, LTA isolated from K8 showed the lowest binding affinity to TLR4. The binding affinity to CD14 was highest with LPS and lowest 
with LTA isolated from K8. This indicates a strong association between TLR4 and CD14 in LPS. Similarly, LTAs isolated from K5-5 and K55-5 appeared to induce stronger signaling than LTAs isolated from K8 and K88 through association with TLR2 and CD14.
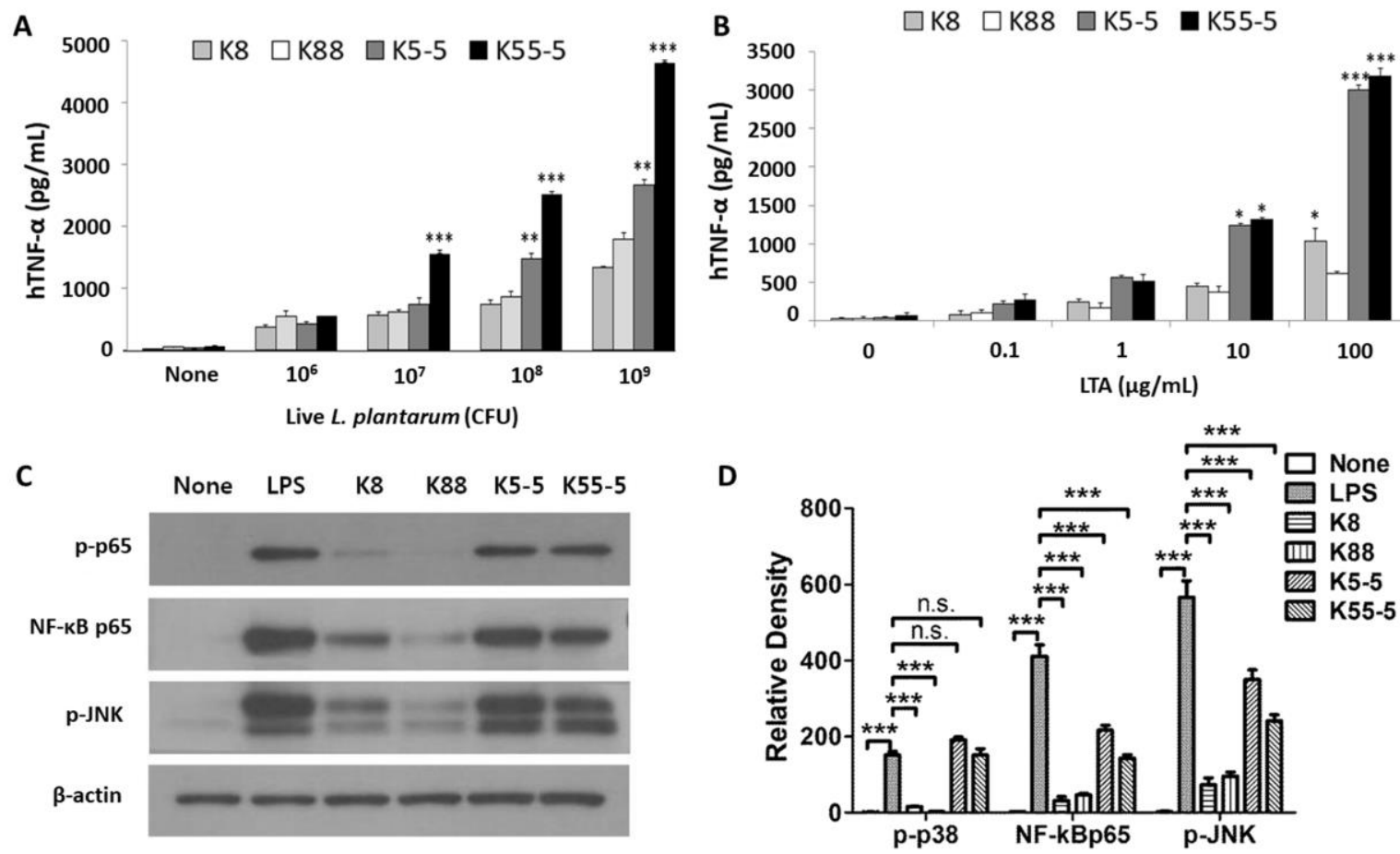

Figure 2. Different immune activities according to strain. (A) TNF- $\alpha$ expression level by four strains of L. plantarum live cells. (B) TNF- $\alpha$ expression level by LTAs isolated from four strains of $L$. plantarum. (C) The activation of NF-kB-p65, p38, and JNK signals was examined by Western Blot. (D) Density analysis of Western blots was performed with ImageJ software (National Institutes of Health, Bethesda, MD, USA). Statistical analysis of the experimental data was performed using one-way analysis of variance (ANOVA), followed by Tukey's honestly significant difference (HSD) post hoc test $(\mathbf{A}, \mathbf{B})$ and two-way ANOVA (D). ${ }^{*} p<0.05$; ${ }^{* *} p<0.01$; and ${ }^{* * *} p<0.001$ as compared to none. n.s. means not significant.

Table 1. Analysis of interaction affinity between LTAs isolated from four strains of L. plantarum and receptors.

\begin{tabular}{|c|c|c|c|c|c|c|c|c|c|}
\hline \multirow[b]{2}{*}{ Analytes } & \multicolumn{3}{|c|}{ TLR2 } & \multicolumn{3}{|c|}{ TLR4 } & \multicolumn{3}{|c|}{ CD14 } \\
\hline & $\begin{array}{c}\mathbf{K a} \\
\left(\mathbf{M}^{-1} \mathbf{S}^{-1}\right)\end{array}$ & $\mathrm{Kd}\left(\mathrm{S}^{-1}\right)$ & $\begin{array}{l}\text { KD } \\
(\mathrm{nM})\end{array}$ & $\begin{array}{c}\mathrm{Ka} \\
\left(\mathbf{M}^{-1} \mathbf{S}^{-1}\right)\end{array}$ & $\mathrm{Kd}\left(\mathrm{S}^{-1}\right)$ & $\begin{array}{l}\text { KD } \\
(\mathrm{nM})\end{array}$ & $\begin{array}{c}\mathbf{K a} \\
\left(M^{-1} S^{-1}\right)\end{array}$ & $\mathrm{Kd}\left(\mathrm{S}^{-1}\right)$ & $\begin{array}{l}\text { KD } \\
\text { (nM) }\end{array}$ \\
\hline LPS & $4.58 \times 10^{4}$ & $3.65 \times 10^{-4}$ & 7.97 & $6.93 \times 10^{4}$ & $2.08 \times 10^{-4}$ & 30.1 & $3.71 \times 10^{4}$ & $2.36 \times 10^{-4}$ & 6.36 \\
\hline K8 & $9.50 \times 10^{3}$ & $5.90 \times 10^{-4}$ & 62.1 & $8.98 \times 10^{3}$ & $6.20 \times 10^{-4}$ & 69.0 & $1.09 \times 10^{4}$ & $3.37 \times 10^{-4}$ & 30.8 \\
\hline K88 & $1.20 \times 10^{4}$ & $5.80 \times 10^{-4}$ & 48.3 & $1.15 \times 10^{4}$ & $5.90 \times 10^{-4}$ & 51.3 & $1.30 \times 10^{4}$ & $3.27 \times 10^{-4}$ & 25.2 \\
\hline K5-5 & $2.00 \times 10^{4}$ & $5.02 \times 10^{-4}$ & 25.1 & $1.61 \times 10^{4}$ & $6.00 \times 10^{-4}$ & 37.2 & $1.83 \times 10^{4}$ & $2.76 \times 10^{-4}$ & 15.09 \\
\hline K55-5 & $3.16 \times 10^{4}$ & $5.84 \times 10^{-4}$ & 18.5 & $2.14 \times 10^{4}$ & $6.51 \times 10^{-4}$ & 30.5 & $1.68 \times 10^{4}$ & $2.04 \times 10^{-4}$ & 12.14 \\
\hline
\end{tabular}

\subsection{Analysis of the LTA Structure}

LTA is a major component of the cell wall of gram-positive bacteria, and the structure of LTA differs by species. The most common LTA structure consists of a glycolipid anchor fixed to the membrane glycolipid, about 50 repeated polyglycerol phosphate chains, and a sugar moiety connecting them [13]. Glyceride (glycerol + fatty acid) from the lipoteichoic acid-like structure is obtained from free fatty acid through alkaline hydrolysis. In addition, the hydroxyl group bonded to the carboxyl group of the fatty acid is methylated to form a 
volatile compound that can be easily analyzed by gas chromatography/mass spectrometry (GC/MS). In total ion chromatogram (TIC) analysis of GC for the four LTAs, the same peaks were detected at RT 14.01 and 16.75 min (Figure 3A). Further, for the three LTAs, K8, $\mathrm{K} 88$, and $\mathrm{K} 55-5$, the same peak was detected at RT $15.2 \mathrm{~min}$, while a peak at 15.86 min was produced for K8 and K88 LTAs, but not for K55-5. These results show that the compounds have a similar structure.

A

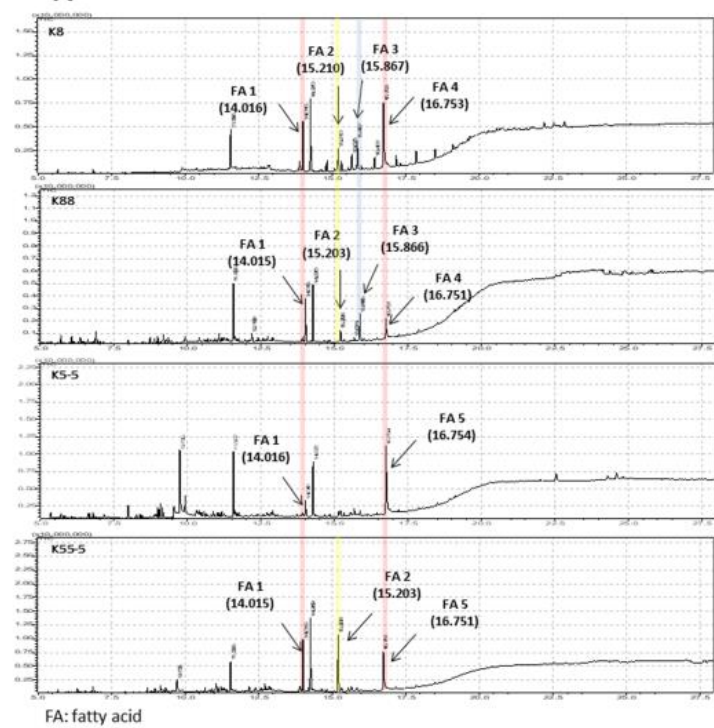

B

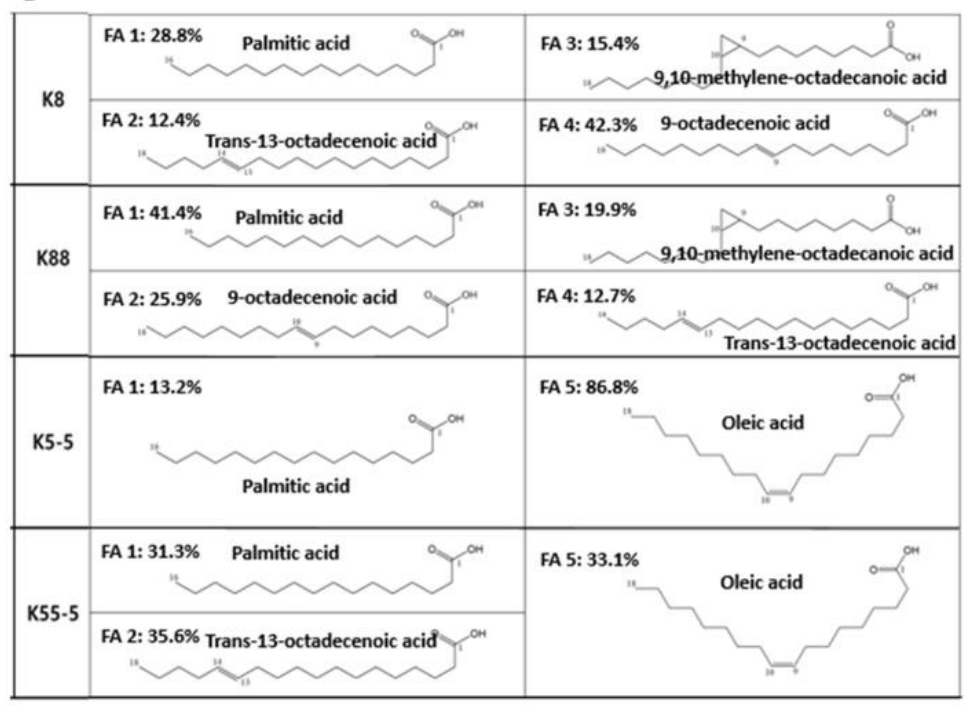

Figure 3. Structural analysis of four LTAs. (A) GC-MS analysis of four LTAs. (B) Composition and content of LTA glycolipids predicted by GC-MS analysis.

The structure was confirmed using the $\mathrm{m} / \mathrm{z}$ value and the DB library based on the mass spectrum, which confirmed the structure shown in Figure 3B. The proportion of each fatty acid present in the structure was determined according to peak area. Additionally, structural analysis of the four types of LTAs was performed using liquid chromatography (LC)-MS. Peak patterns grouped by strains of K8, K88, K5-5, and K55-5 were confirmed. Based on this, the structure of each fatty acid was analyzed and the difference in structural material predicted by the fatty acid structure was confirmed. LC-MS structural analysis showed a difference in the content of LTA types. LTAs of K8 and K88 showed a very similar pattern and a somewhat similar pattern to that of K5-5, but the K55-5 LTA showed a markedly different pattern from the other LTAs (Figure 3B).

\subsection{Suggested Glycolipid Structures of the Four LTAs}

The structures of LTA indicator components one and two were modified according to the structure on GC-MS and reference studies on the existing structure (Figure 4A). The peak in the 23.6 segment was significantly decreased and the peak in the segment 22.22 was greatly increased in K55-5 LTA. In total, 12 peaks, including two main components of LTA, were identified, and these peaks are related according to the numbers of sugars and double bonds in the lipid chain. Two of the LTA components were selected as markers based on LC-MS analysis. LTA marker one appeared at $22.7 \mathrm{~min}$ of RT, and LTA marker two appeared at 23.59 min of RT (Table 2). LTA markers one and two were abundant in the four types of LTA. It is expected that the LTA markers selected in this study can be used as indicators to quantify LTA and maintain quality. Based on the analysis of GC-MS and LC-MS, we predicted the structure of LTA glycolipids (Figure 4B). The proposed glycolipid structural material was expected to act as a substitute for LTA, which is expected to be a major immune-active material of lactic acid bacteria. 
A

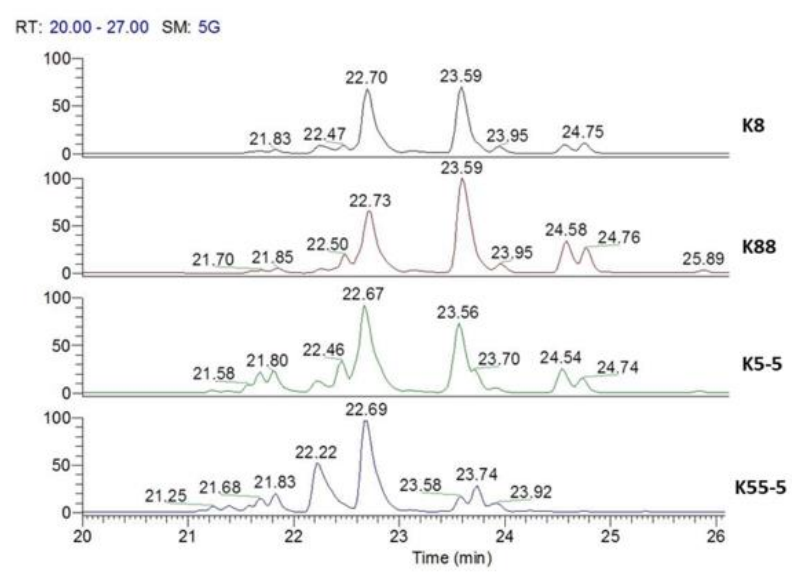

B

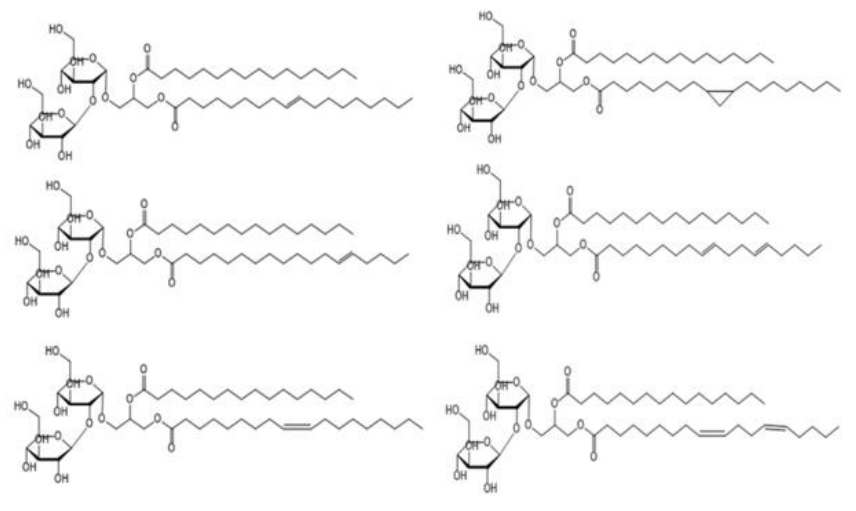

Figure 4. Predicted LTA glycolipid structures for derivatization. (A) LC-MS analysis of four LTAs. (B) Predicted LTA glycolipid structures for derivatization.

Table 2. LC-MS analysis. Relative peak area of two markers isolated from four kinds of LTAs.

\begin{tabular}{|c|c|c|c|c|c|c|c|c|c|}
\hline \multirow{2}{*}{ Target } & & \multirow{2}{*}{ RT (min) } & \multirow{2}{*}{$\begin{array}{c}\mathrm{m} / \mathbf{z} \\
\left((\mathrm{M}+\mathrm{FA}-\mathrm{H})^{-}\right)\end{array}$} & \multirow{2}{*}{ Formula } & \multirow{2}{*}{$\Delta$ ppm } & \multicolumn{4}{|c|}{ Area (\%) } \\
\hline & & & & & & K8 & K88 & K5-5 & K55-5 \\
\hline \multirow{6}{*}{963.63} & sugar, reduction & $21.26 / 21.40$ & 1123.6630 & $\mathrm{C}_{55} \mathrm{H}_{98} \mathrm{O}_{20}$ & 0.533 & 0.6 & 0.3 & 1.5 & 4.4 \\
\hline & reduction & $21.71 / 21.83$ & 961.6102 & $\mathrm{C}_{49} \mathrm{H}_{88} \mathrm{O}_{15}$ & 0.855 & 2.9 & 2.7 & 10.4 & 9.6 \\
\hline & +sugar & 22.26 & 1125.6788 & $\mathrm{C}_{55} \mathrm{H}_{100} \mathrm{O}_{20}$ & 0.621 & 6.9 & 2.6 & 5.3 & 26.9 \\
\hline & LTA Marker 1 & 22.7 & 963.6263 & $\mathrm{C}_{49} \mathrm{H}_{90} \mathrm{O}_{15}$ & 1.497 & 35.1 & 26.4 & 31.4 & 40.2 \\
\hline & reduction-sugar & $22.59 / 22.77$ & 799.5579 & $\mathrm{C}_{43} \mathrm{H}_{78} \mathrm{O}_{10}$ & 1.571 & 0.2 & 0.2 & 0.7 & 0.8 \\
\hline & -sugar & 23.73 & 801.5728 & $\mathrm{C}_{43} \mathrm{H}_{80} \mathrm{O}_{10}$ & 0.731 & 4.4 & 3.3 & 6.0 & 8.5 \\
\hline \multirow{6}{*}{977.64} & sugar, reduction & $22.04 / 22.16$ & 1137.6783 & $\mathrm{C}_{56} \mathrm{H}_{100} \mathrm{O}_{20}$ & 0.386 & 0.7 & 0.7 & 1.2 & 0.4 \\
\hline & reduction & $22.47 / 22.62$ & 975.6256 & $\mathrm{C}_{50} \mathrm{H}_{90} \mathrm{O}_{15}$ & 0.525 & 6.4 & 11.3 & 13.7 & 0.9 \\
\hline & +sugar & 23.11 & 1139.6940 & $\mathrm{C}_{56} \mathrm{H}_{102} \mathrm{O}_{20}$ & 0.403 & 3.6 & 2.4 & 1.6 & 2.1 \\
\hline & LTA Marker 2 & 23.59 & 977.6420 & $\mathrm{C}_{50} \mathrm{H}_{92} \mathrm{O}_{15}$ & 1.342 & 33.8 & 41.1 & 22.9 & 5.4 \\
\hline & reduction-sugar & $23.47 / 23.64$ & 813.5734 & $\mathrm{C}_{44} \mathrm{H}_{80} \mathrm{O}_{10}$ & 1.359 & 0.5 & 0.8 & 1.3 & 0.1 \\
\hline & -sugar & 24.75 & 815.5881 & $\mathrm{C}_{44} \mathrm{H}_{82} \mathrm{O}_{10}$ & 0.24 & 4.7 & 8.3 & 4.0 & 0.7 \\
\hline
\end{tabular}

\section{Discussion}

Lactobacillus species are used as probiotics in humans [14]. For example, L. plantarum reduces serum cholesterol, LDL-cholesterol, and triglyceride levels [15] and attenuates inflammatory bowel disease [16]. L. rhamnosus suppresses allergic sensitization and airway inflammation [17]. L. delbrueckii can protect against Listeria monocytogenes [18], and L. sakei has been suggested to have beneficial effects in children with atopic eczema-dermatitis syndrome [15]. The survival rate of L. acidophilus LA-5, which was microencapsulated in sodium alginate followed by a fish gelatin coating, was improved during baking and storage, suggesting that it can be used as an effective bread enhancer [19]. The immunostimulating potency of lactobacilli appears to be species-specific [20]. For example, a previous study reported that L. rhamnosus and L. plantarum moderately induced the expression of co-stimulatory molecules and stimulated low-level production of cytokines and chemokines [21]. In contrast, $L$. delbrueckii was reported to stimulate the production of high levels of the pro- and anti-inflammatory cytokines TNF- $\alpha$ and IL-10, respectively, as well as that of NO in the mucosal immune system [18]. 
Although $16 \mathrm{~S}$ rRNA sequence analysis of four strains of L. plantarum showed the phylogenetic relationships between reported L. plantarum and the four strains used in this study, the LTA composition in the cell wall and immunoregulatory differences of LTAs were not identical to each other. The composition and structure of LTA seems to be determined at the genetic level [22]. The various activities of probiotics are often classified according to the characteristics of the strain [23]. Strains of the same species are expected to have similar abilities. However, through this study, we confirmed that even the same species can have different activities, and the activity is related to the structural differences of LTAs. Similar studies have been reported. When comparing the effect of a lactobacillus genus on immune action, the degree of induced inflammatory response was dependent on the level of MAPK activity [4]. Strain-specific structural differences in LTA led to differences in binding capacity with its receptors, which led to continuity or strength of the signal pathway; a strong signal eventually produced immune activation. Regarding the activity and association of the two grouped L. plantarum strains, there are likely several structural differences in the binding capacity of LTA and its receptor. However, it is expected that the number of fatty acids in the glycolipid and the number of double bonds in the lipid chain had the largest effect. The double bonds of the lipid chain are expected to be involved in generation of immune substances, as well as with signal activity, as they act on strong binding to receptors such as TLRs [24-26]. It is difficult to see the LTA component of gram-positive bacteria as a single substance. However, the composition and structural differences of the active material act directly.

Analysis of SPR using LTAs and cell surface receptors showed faster and stronger association with ligands (TLR2, TLR4, and CD14) with the same pattern as that of the signal activity $[11,27]$. In addition, the affinity also was revealed to be high in the same pattern through the analyzed KD index. Through this analysis, we determined that the differences in binding ability with receptors of LTA, a component of the gram-positive bacterial cell wall, directly affect signal activity, which leads to a difference in the expression of immune activity downstream. Indeed, SPR analysis showed that LTAs isolated from K5-5 and K55-5 induced stronger signaling than LTAs isolated from K8 and K88 through association with TLR2 and CD14. The various affinity results of LTAs for TLR2 and CD14 appear to be caused by structural differences in LTAs. Therefore, we separated the glycolipids of the four LTAs, analyzed the distribution of the stroma composing the LTA through GC-MS and LC-MS analyses, and predicted the structures of the four LTAs. All four LTAs contained palmitic acid. In addition to palmitic acid, the K8 and K88 LTAs were composed of 9-octadecenoci acid, 9,10-methylene-octadecanoate, and trans-13-octadecenoic acid. On the other hand, the K5-5 and K55-5 LTAs contained a large amount of oleic acid, in addition to palmitic acid. In this way, not only did the strains contain both common and differing substances, but their retention rates were also different depending on LTAs. This shows that the structure depends on the constituents and their amounts affecting the binding ability with TLR2 and CD14. This is attributed to the difference in the initiation and outcome of signal transduction.

In conclusion, through this study, the immune differences of the same bacterial species with different strain number were analyzed using their LTAs. The different binding abilities with the host cell surface receptors, along with the differences in signal pathway, were due to the structural differences in LTAs. LTA is proposed as a major material with multiple functions in lactic acid bacteria. In addition, the structure of a new substance (glycolipid) that can be used in various industrial methods is presented. If this glycolipid can be synthesized as a substitute for lactic acid bacteria, many formulation limitations can be overcome, and the material will be industrially applicable in a variety of fields.

Supplementary Materials: The following supporting information can be downloaded at: https: / / www.mdpi.com/article/10.3390/app12030954/s1. Figure S1: Extended phylogenetic trees of four stains of Lactobacillus plantarum; Figure S2: SPR analysis using LTAs and TLR2, TLR4, and CD14. 
Author Contributions: B.-J.J. and D.-K.C. conceived and planned the experiments; B.-J.J. and H.K. wrote the manuscript; B.-J.J. carried out the experiments; H.K. and D.-K.C. contributed to the interpretation of the results; D.-K.C. supervised the project. All authors have read and agreed to the published version of the manuscript.

Funding: This research was financially supported by the Ministry of Trade, Industry, and Energy (MOTIE), Korea, under the "Infrastructure Support Program for Industry Innovation"(P114000049) supervised by the Korea Institute for Advancement of Technology (KIAT).

Institutional Review Board Statement: Not applicable.

Informed Consent Statement: Not applicable.

Data Availability Statement: The data presented in this study are available in the article.

Conflicts of Interest: The authors declare no conflict of interest.

\section{References}

1. Azad, M.A.K.; Sarker, M.; Li, T.; Yin, J. Probiotic Species in the Modulation of Gut Microbiota: An Overview. Biomed. Res. Int. 2018, 2018, 9478630. [CrossRef]

2. Chapot-Chartier, M.P.; Kulakauskas, S. Cell wall structure and function in lactic acid bacteria. Microb. Cell Fact. 2014, 13, S9. [CrossRef]

3. Leemans, J.C.; Vervoordeldonk, M.J.B.M.; Florquin, S.; van Kessel, K.P.; van der Poll, T. Differential role of interleukin-6 in lung inflammation induced by lipoteichoic acid and peptidoglycan from Staphylococcus aureus. Am. J. Respir. Crit. Care Med. 2002, 165, 1445-1450. [CrossRef]

4. Jeong, J.H.; Jang, S.; Jung, B.J.; Jang, K.S.; Kim, B.G.; Chung, D.K.; Kim, H. Differential immune-stimulatory effects of LTAs from different lactic acid bacteria via MAPK signaling pathway in RAW 264.7 cells. Immunobiology 2015, 220, 460-466. [CrossRef]

5. De Kimpe, S.J.; Kengatharan, M.; Thiemermann, C.; Vane, J.R. The cell wall components peptidoglycan and lipoteichoic acid from Staphylococcus aureus act in synergy to cause shock and multiple organ failure. Proc. Natl. Acad. Sci. USA 1995, 92, 10359-10363. [CrossRef]

6. $\quad$ Kim, H.G.; Kim, N.R.; Gim, M.G.; Lee, J.M.; Lee, S.Y.; Ko, M.Y.; Kim, J.Y.; Han, S.H.; Chung, D.K. Lipoteichoic acid isolated from Lactobacillus plantarum inhibits lipopolysaccharide-induced TNF-alpha production in THP-1 cells and endotoxin shock in mice. J. Immunol. 2008, 180, 2553-2561. [CrossRef]

7. Kim, H.G.; Lee, S.Y.; Kim, N.R.; Ko, M.Y.; Lee, J.M.; Yi, T.H.; Chung, S.K.; Chung, D.K. Inhibitory effects of Lactobacillus plantarum lipoteichoic acid (LTA) on Staphylococcus aureus LTA-induced tumor necrosis factor-alpha production. J. Microbiol. Biotechnol. 2008, 18, 1191-1196.

8. Pan, Z.K. Toll-like receptors and TLR-mediated signaling: More questions than answers. Am. J. Physiol. Lung Cell Mol. Physiol. 2004, 286, L918-L920. [CrossRef]

9. You, L.; Kruse, F.E.; Bacher, S.; Schmitz, M.L. Lipoteichoic acid selectively induces the ERK signaling pathway in the cornea. Invest. Ophthalmol. Vis. Sci. 2002, 43, 2272-2277.

10. Hsieh, H.L.; Lin, C.C.; Shih, R.H.; Hsiao, L.D.; Yang, C.M. NADPH oxidase-mediated redox signal contributes to lipoteichoic acid-induced MMP-9 upregulation in brain astrocytes. J. Neuroinflamm. 2012, 9, 110. [CrossRef]

11. Kwak, M.S.; Lim, M.; Lee, Y.J.; Lee, H.S.; Kim, Y.H.; Youn, J.H.; Choi, J.E.; Shin, J.S. HMGB1 Binds to Lipoteichoic Acid and Enhances TNF- $\alpha$ and IL-6 Production through HMGB1-Mediated Transfer of Lipoteichoic Acid to CD14 and TLR2. J. Innate Immun. 2015, 7, 405-416. [CrossRef]

12. Jang, K.S.; Baik, J.E.; Han, S.H.; Chung, D.K.; Kim, B.G. Multi-spectrometric analyses of lipoteichoic acids isolated from Lactobacillus plantarum. Biochem. Biophys. Res. Commun. 2011, 407, 823-830. [CrossRef]

13. Morath, S.; von Aulock, S.; Hartung, T. Structure/function relationships of lipoteichoic acids. J. Endotoxin Res. 2005, 11, 348-356. [CrossRef]

14. Parvez, S.; Malik, K.A.; Ah Kang, S.; Kim, H.Y. Probiotics and their fermented food products are beneficial for health. J. Appl. Microbiol. 2006, 100, 1171-1185. [CrossRef]

15. Xie, N.; Cui, Y.; Yin, Y.N.; Zhao, X.; Yang, J.W.; Wang, Z.G.; Fu, N.; Tang, Y.; Wang, X.H.; Liu, X.W. Effects of two Lactobacillus strains on lipid metabolism and intestinal microflora in rats fed a high-cholesterol diet. BMC Complement. Altern. Med. $2011,11,53$.

16. Schultz, M.; Veltkamp, C.; Dieleman, L.A.; Grenther, W.B.; Wyrick, P.B.; Wyrick, P.B.; Tonkonogy, S.L.; Sartor, R.B. Lactobacillus plantarum $299 \mathrm{~V}$ in the treatment and prevention of spontaneous colitis in interleukin-10-deficient mice. Inflamm. Bowel Dis. 2002, 8, 71-80. [CrossRef] [PubMed]

17. Feleszko, W.; Jaworska, J.; Rha, R.D.; Steinhausen, S.; Avagyan, A.; Jaudszus, A.; Ahrens, B.; Groneberg, D.A.; Wahn, U.; Hamelmann, E. Probiotic-induced suppression of allergic sensitization and airway inflammation is associated with an increase of T regulatory-dependent mechanisms in a murine model of asthma. Clin. Exp. Allergy 2007, 37, 498-505. [CrossRef] 
18. dos Santos, L.M.; Santos, M.M.; de Souza Silva, H.P.; Arantes, R.M.; Nicoli, J.R.; Vieira, L.Q. Monoassociation with probiotic Lactobacillus delbrueckii UFV-H2b20 stimulates the immune system and protects germfree mice against Listeria monocytogenes infection. Med. Microbiol. Immunol. 2011, 200, 29-38. [CrossRef]

19. Hadidi, M.; Majidiyan, N.; Jelyani, A.Z.; Moreno, A.; Hadian, Z.; Mousavi Khanegah, A. Alginate/Fish Gelatin-Encapsulated Lactobacillus acidophilus: A Study on Viability and Technological Quality of Bread during Baking and Storage. Foods 2021, 10, 2215. [CrossRef]

20. Ryu, Y.H.; Baik, J.E.; Yang, J.S.; Kang, S.S.; Im, J.; Yun, C.H.; Kim, D.W.; Lee, K.; Chung, D.K.; Ju, H.R. Differential immunestimulatory effects of gram-positive bacteria due to their lipoteichoic acids. Int. Immunopharmacol. 2009, 9, 127-133. [CrossRef]

21. Ding, Y.; Qian, L.; Pang, J.; Lin, J.; Xu, Q.; Wang, L.; Huang, D.; Zou, H. The regulation of immune cells by Lactobacilli: A potential therapeutic target for anti-atherosclerosis therapy. Oncotarget 2017, 8, 59915-59928. [CrossRef]

22. Reichmann, N.T.; Gründling, A. Location, synthesis and function of glycolipids and polyglycerolphosphate lipoteichoic acid in Gram-positive bacteria of the phylum Firmicutes. FEMS Microbiol. Lett. 2011, 319, 97-105. [CrossRef] [PubMed]

23. Tomás, C.C.; Oliveira, E.; Sousa, D.; Uba-Chupel, M.; Furtado, G.; Rocha, C.; Teixeira, A.; Ferreira, P.; Alves, C.; Gisin, S.; et al. Proceedings of the 3rd IPLeiria's International Health Congress. BMC Health Serv. Res. 2016, 16, 200.

24. Lee, J.Y.; Zhao, L.; Youn, H.S.; Weatherill, A.R.; Tapping, R.; Feng, L.; Lee, W.H.; Fitzgerald, K.A.; Hwang, D.H. Saturated fatty acid activates but polyunsaturated fatty acid inhibits Toll-like receptor 2 dimerized with Toll-like receptor 6 or 1 . J. Biol. Chem. 2004, 279, 16971-16979. [CrossRef] [PubMed]

25. Huang, S.; Rutkowsky, J.M.; Snodgrass, R.G.; Ono-Moore, K.D.; Schneider, D.A.; Newman, J.W.; Adams, S.H.; Hwang, D.H. Saturated fatty acids activate TLR-mediated proinflammatory signaling pathways. J. Lipid Res. 2012, 53, 2002-2013. [CrossRef] [PubMed]

26. Hwang, D.H.; Kim, J.A.; Lee, J.Y. Mechanisms for the activation of Toll-like receptor $2 / 4$ by saturated fatty acids and inhibition by docosahexaenoic acid. Eur. J. Pharmacol. 2016, 785, 24-35. [CrossRef] [PubMed]

27. Shen, Y.; Boulos, S.; Sumrall, E.; Gerber, B.; Julian-Rodero, A.; Eugster, M.R.; Fieseler, L.; Nyström, L.; Ebert, M.O.; Loessner, M.J Structural and functional diversity in Listeria cell wall teichoic acids. J. Biol. Chem. 2017, 292, 17832-17844. [CrossRef] [PubMed] 